\title{
SUBGEOMETRIC RATES OF CONVERGENCE FOR A CLASS OF CONTINUOUS-TIME MARKOV PROCESS
}

\author{
ZHENTING HOU ${ }^{* * *}$ AND \\ YUANYUAN LIU, ${ }^{* * * *}$ Central South University \\ HANJUN ZHANG, ${ }^{* * * *}$ University of Queensland
}

\begin{abstract}
Let $\left(\Phi_{t}\right)_{t \in \mathbb{R}_{+}}$be a Harris ergodic continuous-time Markov process on a general state space, with invariant probability measure $\pi$. We investigate the rates of convergence of the transition function $P^{t}(x, \cdot)$ to $\pi$; specifically, we find conditions under which $r(t)\left\|P^{t}(x, \cdot)-\pi\right\| \rightarrow 0$ as $t \rightarrow \infty$, for suitable subgeometric rate functions $r(t)$, where $\|\cdot\|$ denotes the usual total variation norm for a signed measure. We derive sufficient conditions for the convergence to hold, in terms of the existence of suitable points on which the first hitting time moments are bounded. In particular, for stochastically ordered Markov processes, explicit bounds on subgeometric rates of convergence are obtained. These results are illustrated in several examples.
\end{abstract}

Keywords: Continuous-time Markov process; queueing model; birth-death process; ergodicity; subgeometric convergence

2000 Mathematics Subject Classification: Primary 60J25

Secondary $60 \mathrm{~K} 25$

\section{Introduction}

In the past few decades, much effort has been made to study ergodic theory for continuoustime Markov processes. To the authors' knowledge, the literature (e.g. [1], [3], [13], and [14]) has focused on Harris ergodicity, exponential ergodicity, and strong ergodicity. For discrete-time Markov processes, there has been much work (e.g. [15] and [18]) on subgeometric ergodicity, which is, roughly speaking, a kind of convergence quicker than ordinary ergodicity and slower than exponential convergence. However, there are few works on subgeometric convergence for continuous-time Markov processes, the topic of this paper. To obtain our results, we shall make the following assumption about the Markov process.

Assumption 1.1. There exists a state $x_{0}$ such that whenever the Markov process hits $x_{0}$, it will sojourn there for a random time that is positive with probability 1.

It is easy to find many Markov processes satisfying Assumption 1.1, for example many queueing processes and all the totally stable continuous-time Markov chains (i.e. continuoustime Markov processes on countable state spaces). If a continuous-time Markov process satisfies

Received 11 October 2004; revision received 2 February 2005.

* Postal address: School of Mathematics, Central South University, Changsha, Hunan, 410075, P. R. China.

** Email address: zthou@csu.edu.cn

*** Email address: liuyy@csu.edu.cn

**** Postal address: Department of Mathematics, The University of Queensland, Queensland, 4072, Australia. Email address: hjz@maths.uq.edu.au 
Assumption 1.1 then, due to the Markov property and the homogeneity of the process, it can be easily proved that the sojourn time in state $x_{0}$ is exponentially distributed with some parameter $\lambda$, $0<\lambda<\infty$. Thus, the state $x_{0}$ behaves just like a state in a continuous-time Markov chain, and we can apply the skeleton chain method, used to study continuous-time Markov chains, to the continuous-time Markov process.

Throughout the paper, we denote by $\mathbb{R}_{+}$the set of nonnegative real numbers, by $\mathbb{Z}_{+}$the set of nonnegative integers, and by $\mathbb{N}_{+}$the set of positive integers. Let $\left(\Phi_{t}\right)_{t \in \mathbb{R}_{+}}$be a timehomogeneous continuous-time Markov process satisfying Assumption 1.1 and taking values in a general state space $X$ endowed with a countably generated $\sigma$-field $\mathscr{B}(X)$. We denote by $P^{t}(x, A), t \in \mathbb{R}_{+}, A \in \mathscr{B}(X)$, the transition function of the Markov process:

$$
P^{t}(x, A)=\mathrm{P}_{x}\left[\Phi_{t} \in A\right]=\mathrm{E}_{x}\left[\mathbf{1}_{\left\{\Phi_{t} \in A\right\}}\right] .
$$

Here, $\mathrm{P}_{x}$ and $\mathrm{E}_{x}$ respectively denote the probability and expectation of the Markov process under the initial condition $\Phi_{0}=x$, and $\mathbf{1}_{\{\cdot\}}$ is an indicator function.

In order to study the subgeometric rates of convergence, we suppose that $\Phi_{t}$ is Harris ergodic with unique invariant probability measure $\pi$, i.e. for all $x \in X$,

$$
\left\|P^{t}(x, \cdot)-\pi\right\| \rightarrow 0, \quad t \rightarrow \infty,
$$

where $\|\mu\|=\sup _{|g| \leq 1}|\mu(g)|$ denotes the usual total variation norm for a signed measure $\mu$. Obviously, if $\Phi_{t}$ is Harris ergodic then it is $\varphi$-irreducible and Harris recurrent.

Now we introduce a collection of subgeometric rate functions that includes, for example, all functions of the form $r(t)=t^{\alpha}, \alpha>0$. Here, $r(t)$ is a continuous-time version of $r(n)$ in [18], originally introduced in [16]. To define this class, we first denote by $\Lambda_{0}$ the family of those functions $r:(0, \infty) \rightarrow(0, \infty)$ such that $r(t)$ is continuous and increasing in $t$ with $r(1) \geq 2$ and

$$
\frac{\log r(t)}{t} \downarrow 0 \quad \text { as } t \uparrow \infty .
$$

Denote by $\Lambda$ those functions $r$ for which both $r(t)>0$ for all $t \in \mathbb{R}_{+}$and there exists an $r_{0} \in \Lambda_{0}$ which is equivalent to $r$ in the sense that

$$
\liminf _{t \rightarrow \infty} \frac{r(t)}{r_{0}(t)}>0 \text { and } \quad \limsup _{t \rightarrow \infty} \frac{r(t)}{r_{0}(t)}<\infty .
$$

Without loss of generality, we assume that $r(0)<1$ whenever $r \in \Lambda$. The properties of these functions $r \in \Lambda_{0}$ that we will use most frequently below, which follow from (1.2), are

$$
\begin{aligned}
& r(x+y) \leq r(x) r(y) \quad \text { for all } x, y \in[0, \infty), \\
& \frac{r(x+a)}{r(x)} \rightarrow 1 \quad \text { as } x \rightarrow \infty, \quad \text { for each } a \in(0, \infty) .
\end{aligned}
$$

The structure of the paper is as follows. In Section 2, we obtain the main result, finding conditions under which the subgeometric convergence

$$
r(t)\left\|P^{t}(x, \cdot)-\pi\right\| \rightarrow 0, \quad t \rightarrow \infty,
$$

holds for $\pi$-almost every ( $\pi$-a.e.) $x \in X$, where $r(t)$ is a subgeometric rate function. In Section 3, this result is applied to the queue length process of the M/G/1 queue with multiple vacations. In particular, when the Markov processes are stochastically ordered, we are interested 
in obtaining more explicit results for the subgeometric rates of convergence, i.e. in finding explicit bounds on (a bounding function) $M(x)$ such that

$$
\int_{0}^{\infty} r(t)\left\|P^{t}(x, \cdot)-\pi\right\| \mathrm{d} t \leq M(x)
$$

for $r \in \Lambda$ and every $x \in X$. In Section 4, we apply the coupling method to obtain explicit bounds on $M(x)$. In Section 5 and Section 6, we discuss the M/G/1 queue and continuous-time birth-death chains, respectively - two stochastically monotone examples in which computable bounds on $M(x)$ are given.

\section{Subgeometric rates of convergence}

Let $\left(\Phi_{n h}\right)_{n \in \mathbb{Z}_{+}}, h \in \mathbb{R}_{+}$, be a skeleton chain of the Markov process $\left(\Phi_{t}\right)_{t \in \mathbb{R}_{+}}$. Theorem 1 of [17] states that if any skeleton chain $\Phi_{n h}$ is Harris ergodic, then so is $\Phi_{t}$. In fact, if $\Phi_{t}$ is Harris ergodic, by setting $t=n h$ in (1.1) we have $\left\|P^{n h}(x, \cdot)-\pi\right\| \rightarrow 0, n \rightarrow \infty$, i.e. the skeleton chain $\Phi_{n h}$ is also Harris ergodic. The following lemma shows the equivalence between the subgeometric convergences of $\Phi_{n h}$ and $\Phi_{t}$.

Lemma 2.1. A Markov process $\Phi_{t}$ is subgeometrically convergent if and only if so too is any skeleton chain $\Phi_{n h}$ of $\Phi_{t}$.

Proof. If $\Phi_{t}$ is subgeometrically convergent then, by setting $t=n h$ in (1.5), we know that $\Phi_{n h}$ is also subgeometrically convergent.

For any $h>0$, suppose that $\Phi_{n h}$ is subgeometrically convergent with invariant probability measure $\pi$, i.e. (1.5) holds for $t=n h$. By virtue of Theorem 1 of [17], we know that, for every bounded signed measure $v$ on $(X, \mathscr{B}(X))$, the function $t \mapsto\left\|v P^{t}\right\|$ is decreasing and $\pi$ is the unique invariant probability measure for $P^{t}$. Choose a $t>0$ such that $(k-1) h \leq t<k h$ for $k \in \mathbb{Z}_{+}$. By use of monotonicity and (1.3), we have

$$
\begin{aligned}
r(t)\left\|\mu P^{t}(x, \cdot)-\pi\right\| & =r(t)\left\|(\mu-\pi) P^{t}(x, \cdot)\right\| \\
& \leq r(k h)\left\|(\mu-\pi) P^{(k-1) h}(x, \cdot)\right\| \\
& \leq r(h) r((k-1) h)\left\|\mu P^{(k-1) h}(x, \cdot)-\pi\right\| \rightarrow 0, \quad t \rightarrow \infty,
\end{aligned}
$$

for any probability measure $\mu$. In particular, if in $(2.1)$ we let $\mu(\cdot)=\mathbf{1}_{\{x\}}(\cdot)$ be the single-point probability measure, then we recover (1.5).

Now we recall the definition of a small set: a set $C \in \mathscr{B}(X)$ is called a small set if there exists an $n \in \mathbb{N}_{+}$and a nontrivial measure $v_{n}$ on $\mathscr{B}(X)$ such that, for all $x \in C$ and $B \in \mathscr{B}(X)$, we have $P^{n}(x, B) \geq v_{n}(B)$. Also, let $\tau_{B}=\inf \left\{n: \Phi_{n} \in B\right\}, B \in \mathscr{B}(X)$, and $r^{0}(n)=\sum_{k=0}^{n} r(k)$.

The following lemma can be deduced directly from Theorem 2.7 of [15].

Lemma 2.2. Let $\Phi_{n}$ be a Harris ergodic Markov chain on $X$ with invariant probability measure $\pi$. If $B$ is a small set with $\pi(B)>0$ and $\sup _{x \in B} \mathrm{E}_{x}\left[r^{0}\left(\tau_{B}\right)\right]<\infty$, then

$$
r(n)\left\|P^{n}(x, \cdot)-\pi\right\| \rightarrow 0, \quad n \rightarrow \infty,
$$

for $\pi$-a.e. $x \in X$.

Proof. Since $B$ is a small set of the chain $\Phi_{n}$ and $\mathrm{E}_{x}\left[r^{0}\left(\tau_{B}\right)\right]<\infty$, by virtue of Theorem 2.7(i) and Theorem 2.6 of [15] we have $\mathrm{E}_{x}\left[r\left(\tau_{B}\right)\right] \leq \mathrm{E}_{x}\left[r^{0}\left(\tau_{B}\right)\right]<\infty$ for $\pi$-a.e. $x \in X$. 
Let $\lambda(\cdot)=\mathbf{1}_{\{x\}}(\cdot)$ be the single-point probability measure in Theorem 2.7(ii) of [15]. We then find that (2.2) holds for $\pi$-a.e. $x \in X$.

In the following, we will reveal the relationship between the moments of the first hitting time of the continuous-time Markov process $\Phi_{t}$ and those of its skeleton chain $\Phi_{n h}$.

We write $\hat{r}(t)=\int_{0}^{t} r(x) \mathrm{d} x$ and $r^{0}(n h)=\sum_{k=0}^{n} r(k h)$. Recursively define

$$
\delta_{x_{0}}^{n}=\inf \left\{s>\delta_{x_{0}}^{n-1}+J_{1}: \Phi_{t}=x_{0}\right\},
$$

where $\delta_{x_{0}}^{0}=0$ and $J_{1}$ is the first jump time of the Markov process $\Phi_{t}$. Thus, $\delta_{x_{0}}^{n}$ denotes the time of the $n$th return of $\Phi_{t}$ to $x_{0}$. We write $\delta_{x_{0}}=\delta_{x_{0}}^{1}$, i.e. $\delta_{x_{0}}$ is the time of the first return of $\Phi_{t}$ to $x_{0}$ after the first jump. We also define $\delta_{x_{0}}(h)=h \inf \left\{n \geq 1: \Phi_{n h}=x_{0}\right\}$ to be the first hitting time at $x_{0}$ of the skeleton chain $\Phi_{n h}$.

Theorem 2.1. Let $\Phi_{t}$ be a continuous-time Markov process on X satisfying Assumption 1.1 and let $r \in \Lambda$. The following statements are equivalent.

(i) $\mathrm{E}_{x_{0}}\left[\hat{r}\left(\delta_{x_{0}}\right)\right]<\infty$.

(ii) There exists some $h_{0}>0$ such that $\mathrm{E}_{x_{0}}\left[r^{0}\left(\delta_{x_{0}}\left(h_{0}\right)\right)\right]<\infty$.

Proof. Assumption 1.1 implies that the sojourn time in state $x_{0}$ is exponentially distributed with some parameter $\lambda, 0<\lambda<\infty$. Owing to the equivalence between $\Lambda$ and $\Lambda_{0}$, we may suppose that $r \in \Lambda_{0}$.

Suppose that part (ii) holds and define $h_{n}=h_{0} / 2^{n}, n \in \mathbb{Z}_{+}$. Observe that $\delta_{x_{0}}\left(h_{n}\right) \leq \delta_{x_{0}}\left(h_{0}\right)$ for all $n$. By the assumption that $r(0)<1$, we can find an $n$ such that $P^{h_{n}}\left(x_{0}, x_{0}\right) r\left(h_{n}\right)<1$ and $\mathrm{E}_{x_{0}}\left[r^{0}\left(\delta_{x_{0}}\left(h_{n}\right)\right)\right]<\infty$. For the $h_{n}$-skeleton chain, define $J_{1}\left(h_{n}\right)=\inf \left\{m \in \mathbb{Z}_{+}: \Phi_{m h_{n}} \neq \Phi_{0}\right\}$ and $\delta_{x_{0}}^{+}\left(h_{n}\right)=h_{n} \inf \left\{m \geq J_{1}\left(h_{n}\right): \Phi_{m h_{n}}=x_{0}\right\}$. We clearly have

$$
\begin{aligned}
\mathrm{E}_{x_{0}}\left[r^{0}\left(\delta_{x_{0}}^{+}\left(h_{n}\right)\right)\right]= & \sum_{m=1}^{\infty} \mathrm{E}\left[r^{0}\left(\delta_{x_{0}}\left(h_{n}\right)+m h_{n}\right) \mid J_{1}\left(h_{n}\right)=m, \Phi_{0}=x_{0}\right] \\
& \times \mathrm{P}\left[J_{1}\left(h_{n}\right)=m \mid \Phi_{0}=x_{0}\right] \\
& =\sum_{m=1}^{\infty}\left[P^{h_{n}}\left(x_{0}, x_{0}\right)\right]^{m-1} \int_{X \backslash\left\{x_{0}\right\}} P^{h_{n}}\left(x_{0}, \mathrm{~d} y\right) \mathrm{E}_{y}\left[r^{0}\left(\delta_{x_{0}}\left(h_{n}\right)+m h_{n}\right)\right] \\
& \leq \sum_{m=1}^{\infty}\left[P^{h_{n}}\left(x_{0}, x_{0}\right)\right]^{m-1} \frac{\left[r\left(h_{n}\right)\right]^{m+1}}{r\left(h_{n}\right)-1} \mathrm{E}_{x_{0}}\left[r^{0}\left(\delta_{x_{0}}\left(h_{n}\right)\right)\right] \\
& <\infty,
\end{aligned}
$$

which follows from the fact that

$$
r^{0}\left(\delta_{x_{0}}\left(h_{n}\right)+m h_{n}\right) \leq r^{0}\left(\delta_{x_{0}}\left(h_{n}\right)\right)+\sum_{k=1}^{m} r\left(k h_{n}\right) r\left(\delta_{x_{0}}\left(h_{n}\right)\right) \leq r^{0}\left(\delta_{x_{0}}\left(h_{n}\right)\right) \frac{\left[r\left(h_{n}\right)\right]^{m+1}}{r\left(h_{n}\right)-1} .
$$

The statement of part (i) follows from this and the fact that $\delta_{x_{0}} \leq \delta_{x_{0}}^{+}\left(h_{n}\right)$.

Now suppose that part (i) holds. It is possible that the skeleton chain can 'miss' visits of the continuous-time process to $x_{0}$, and so result in $\delta_{x_{0}}<\delta_{x_{0}}(n h)$. Suppose that $\Phi_{0}=x_{0}$, let $D_{k}$ be the $k$ th sojourn time at $x_{0}$, and let $W_{k}$ be the length of the interval between the $k$ th exit from $x_{0}$ and the next visit to $x_{0}$ of the continuous-time Markov process $\Phi_{t}$. Then $\delta_{x_{0}}^{n}=\sum_{k=1}^{n}\left(D_{k}+W_{k}\right)$. 
Note that the $W_{k}$ are independent and that the $D_{k}$ are independent of each other and the $W_{k}$. Moreover, the $D_{k}$ are identically exponentially distributed with parameter $\lambda$.

Define

$$
N=\min \left\{n \geq 1: \text { the } h \text {-skeleton chain is in state } x_{0} \text { during the interval } D_{n}\right\} .
$$

Then $\delta_{x_{0}}(h) \leq \sum_{i=1}^{N-1}\left(D_{i}+W_{i}\right)+h$ and, by using (1.3), we obtain

$$
\begin{aligned}
\hat{r}\left(n h+\sum_{i=1}^{n-1} W_{i}\right)= & \int_{0}^{n h} r(t) \mathrm{d} t+\int_{n h}^{n h+W_{1}} r(t) \mathrm{d} t+\cdots \\
& +\int_{n h+\sum_{i=1}^{k-1} W_{i}}^{n h+\sum_{i=1}^{k} W_{i}} r(t) \mathrm{d} t+\cdots+\int_{n h+\sum_{i=1}^{n-2} W_{i}}^{n h+\sum_{i=1}^{n-1} W_{i}} r(t) \mathrm{d} t \\
= & \int_{0}^{n h} r(t) \mathrm{d} t+\int_{0}^{W_{1}} r(t+n h) \mathrm{d} t+\cdots \\
& +\int_{0}^{W_{k}} r\left(t+n h+\sum_{i=1}^{k-1} W_{i}\right) \mathrm{d} t+\cdots+\int_{0}^{W_{n-1}} r\left(t+n h+\sum_{i=1}^{n-2} W_{i}\right) \mathrm{d} t \\
\leq & \hat{r}(n h)+r(n h) \hat{r}\left(W_{1}\right)+\cdots+\prod_{i=1}^{k-1} r\left(W_{i}\right) r(n h) \hat{r}\left(W_{k}\right) \\
& +\cdots+\prod_{i=1}^{n-2} r\left(W_{i}\right) r(n h) \hat{r}\left(W_{n-1}\right) .
\end{aligned}
$$

Since the $W_{k}$ are independent and identically distributed, by virtue of (2.3) we obtain

$$
\begin{aligned}
& h \mathrm{E}_{x_{0}}\left[r^{0}\left(\delta_{x_{0}}(h)-h\right)\right] \leq \mathrm{E}_{x_{0}}\left[\hat{r}\left(\delta_{x_{0}}(h)\right)\right] \leq \mathrm{E}_{x_{0}}\left[\hat{r}\left(\sum_{i=1}^{N-1}\left(D_{i}+W_{i}\right)+h\right)\right] \\
& =\sum_{n=1}^{\infty} \mathrm{E}_{x_{0}}\left[\hat{r}\left(\sum_{i=1}^{n-1}\left(D_{i}+W_{i}\right)+h\right) \mathbf{1}_{\{N=n\}}\right] \\
& \leq \sum_{n=1}^{\infty} \mathrm{E}_{x_{0}}\left[\hat{r}\left(\sum_{i=1}^{n-1} W_{i}+n h\right) \mathbf{1}_{\bigcap_{k=1}^{n-1}\left\{D_{k} \leq h\right\}}\right] \\
& \leq \sum_{n=1}^{\infty} \mathrm{E}_{x_{0}}\left[\hat{r}\left(n h+\sum_{i=1}^{n-1} W_{i}\right)\right]\left(1-\mathrm{e}^{-\lambda h}\right)^{n-1} \\
& \leq \sum_{n=1}^{\infty}\left(1-\mathrm{e}^{-\lambda h}\right)^{n-1}\left\{\hat{r}(n h)+\mathrm{E}\left[\hat{r}\left(W_{1}\right)\right] r(n h) \sum_{m=0}^{n-2} \mathrm{E}\left[r\left(W_{1}\right)\right]^{m}\right\} \\
& \leq \sum_{n=1}^{\infty}\left(1-\mathrm{e}^{-\lambda h}\right)^{n-1} r(n h) \mathrm{E}\left[\hat{r}\left(W_{1}\right)\right] \frac{\mathrm{E}\left[r\left(W_{1}\right)\right]-\left(\mathrm{E}\left[r\left(W_{1}\right)\right]\right)^{n-1}}{1-\mathrm{E}\left[r\left(W_{1}\right)\right]} \\
& +\sum_{n=1}^{\infty}\left(1-\mathrm{e}^{-\lambda h}\right)^{n-1} \hat{r}(n h) .
\end{aligned}
$$


Now we show that we can choose a suitable $h$ such that $\mathrm{E}_{x_{0}}\left[r^{0}\left(\delta_{x_{0}}\left(h_{0}\right)\right)\right]<\infty$. By using (1.4), we obtain

$$
\lim _{n \rightarrow \infty} \frac{r((n-1) h+h)}{r((n-1) h)} \frac{\left(1-\mathrm{e}^{-\lambda h}\right)^{n}}{\left(1-\mathrm{e}^{-\lambda h}\right)^{n-1}}=1-\mathrm{e}^{-\lambda h} \rightarrow 0 \quad \text { as } h \rightarrow 0
$$

and, by using (1.3), we have

$$
\begin{aligned}
\frac{\left(1-\mathrm{e}^{-\lambda h}\right)^{n}}{\left(1-\mathrm{e}^{-\lambda h}\right)^{n-1}} \frac{\hat{r}(n h)}{\hat{r}((n-1) h)} & \leq\left(1-\mathrm{e}^{-\lambda h}\right) \frac{\hat{r}((n-1) h)+h r(n h)}{\hat{r}((n-1) h)} \\
& \leq\left(1-\mathrm{e}^{-\lambda h}\right)(1+h r(2 h)) .
\end{aligned}
$$

It is obvious that $W_{1}<\delta_{x_{0}}$; hence, $\mathrm{E}\left[\hat{r}\left(W_{1}\right)\right]<\infty$ follows from the fact that $\mathrm{E}_{x_{0}}\left[\hat{r}\left(\delta_{x_{0}}\right)\right]<\infty$. By again using (1.3), we have

$$
\begin{aligned}
\mathrm{E}\left[r\left(W_{1}\right)\right] & \leq \mathrm{E}_{x_{0}}\left[r\left(\delta_{x_{0}}\right)\right] \\
& \leq \mathrm{E}_{x_{0}}\left[r\left(\delta_{x_{0}}-\varepsilon\right) r(\varepsilon)\right] \\
& \leq \frac{r(\varepsilon)}{\varepsilon} \mathrm{E}_{x_{0}}\left[\int_{\delta_{x_{0}}-\varepsilon}^{\delta_{x_{0}}} r(t) \mathrm{d} t\right] \\
& <\frac{r(\varepsilon)}{\varepsilon} \mathrm{E}_{x_{0}}\left[\hat{r}\left(\delta_{x_{0}}\right)\right]<\infty
\end{aligned}
$$

for some positive real number $\varepsilon$. From (2.4)-(2.7), we can choose $h_{0}$ to be sufficiently small that

$$
\mathrm{E}_{x_{0}}\left[r^{0}\left(\delta_{x_{0}}\left(h_{0}\right)\right)\right] \leq\left(1+r\left(h_{0}\right)\right) \mathrm{E}_{x_{0}}\left[r^{0}\left(\delta_{x_{0}}\left(h_{0}\right)-h_{0}\right)\right]<\infty .
$$

Thus, we have proved that part (ii) holds. This completes the proof of the theorem.

We make the following remarks concerning Theorem 2.1.

Remark 2.1. If Assumption 1.1 holds then, by an argument similar to the proof of Theorem 2.1, we can show that $\mathrm{E}_{x_{0}}\left[\delta_{x_{0}}\right]<\infty$ if and only if $\mathrm{E}_{x_{0}}\left[\delta_{x_{0}}\left(h_{0}\right)\right]<\infty$ for some $h_{0}$-skeleton chain.

Remark 2.2. For an ergodic continuous-time Markov chain, if $\mathrm{E}_{j}\left[\hat{r}\left(\delta_{j}\right)\right]<\infty$ for some $j \in X$ then, from Theorem 2.1, we see that $\mathrm{E}_{j}\left[r^{0}\left(\delta_{j}\left(h_{0}\right)\right)\right]<\infty$ for some $h_{0}$-skeleton chain. It follows, from Remark 2.8 of [15], that $\mathrm{E}_{i}\left[r^{0}\left(\delta_{j}\left(h_{0}\right)\right)\right]<\infty$ for all $i, j \in X$. Again by virtue of Theorem 2.1, we have $\mathrm{E}_{i}\left[\hat{r}\left(\delta_{i}\right)\right]<\infty$ for any $i \in X$. When $j$, the initial state of $\Phi_{t}$, does not equal $i$, we have $\delta_{i} \leq \delta_{i}\left(h_{0}\right)$, meaning that, for each $j \neq i$,

$$
\begin{aligned}
\mathrm{E}_{j}\left[\hat{r}\left(\delta_{i}\right)\right] & \leq(1+r(2)) \mathrm{E}_{j}\left[\hat{r}\left(\delta_{i}-1\right)\right] \\
& \leq(1+r(2)) \mathrm{E}_{j}\left[r^{0}\left(\left\lfloor\delta_{i}\right\rfloor\right)\right] \\
& \leq(1+r(2)) \mathrm{E}_{j}\left[r^{0}\left(\delta_{i}\left(h_{0}\right)\right)\right]<\infty,
\end{aligned}
$$

where $\lfloor x\rfloor$ is the integer-part function. Thus, we see that if $\mathrm{E}_{j}\left[\hat{r}\left(\delta_{j}\right)\right]<\infty$ for some $j \in X$, then $\mathrm{E}_{i}\left[\hat{r}\left(\delta_{j}\right)\right]<\infty$ for all $i, j \in X$. In particular, let $r(t)=t^{l-1}$ for $l \in \mathbb{R}_{+}$, with $l \geq 1$. If $\mathrm{E}_{i}\left[\delta_{i}^{l}\right]<\infty$ for some $i \in X$ then $\mathrm{E}_{i}\left[\delta_{j}^{l}\right]<\infty$ for all $i, j \in X$.

We are now in a position to establish our main result, which gives sufficient conditions for the subgeometric convergence of a class of continuous-time Markov process. 
Theorem 2.2. Let $\Phi_{t}$ be a Harris ergodic continuous-time Markov process satisfying Assumption 1.1, with invariant probability measure $\pi$. If $r \in \Lambda$ and $\mathrm{E}_{x_{0}}\left[\hat{r}\left(\delta_{x_{0}}\right)\right]<\infty$, then

$$
r(t)\left\|P^{t}(x, \cdot)-\pi(\cdot)\right\| \rightarrow 0, \quad t \rightarrow \infty,
$$

for $\pi$-a.e. $x \in X$.

Proof. It follows, from Lemma 2.1, that any skeleton chain $\Phi_{n h}$ of $\Phi_{t}$ is Harris ergodic. Since $P^{n h}\left(x_{0}, x_{0}\right) \geq \mathrm{e}^{-\lambda h}$ for any $h>0$, it follows that $x_{0}$ is an atom and the single-point set $\left\{x_{0}\right\}$ is a small set of the skeleton chain $\Phi_{n h}$. Hence, $\pi\left(x_{0}\right)>0$. From Theorem 2.1 and Lemma 2.2, we see that there exists a skeleton chain $\Phi_{n h_{0}}$ that is subgeometrically convergent. The assertion therefore follows from Lemma 2.1.

Remark 2.3. For a continuous-time Markov chain $\Phi_{t}$, the total variation norm is such that

$$
\left\|P^{t}(i, \cdot)-\pi(\cdot)\right\|=\sum_{j \in X}\left|P^{t}(i, j)-\pi(j)\right| .
$$

Hence, if $\mathrm{E}_{j}\left[\hat{r}\left(\delta_{j}\right)\right]<\infty$ for some $j \in X$, by using Theorem 2.2 we see that

$$
r(t)\left|P^{t}(i, j)-\pi(j)\right| \rightarrow 0, \quad t \rightarrow \infty,
$$

for all $i, j \in X$. This result extends Theorem 1.2 of [12], which deals with the special case $r(t)=t^{l}, l \in \mathbb{Z}_{+}$, using a different method.

The following theorem and remark play a crucial role in bounding $M(x)$ in Section 4. The theorem is interesting in its own right and tells us that, under some conditions, the subgeometrically convergent Markov chains do have stationary distributions with subgeometric tails.

Theorem 2.3. Let $\Phi_{t}$ be a Harris ergodic continuous-time Markov process satisfying Assumption 1.1, with invariant probability measure $\pi$. If $r \in \Lambda$ and $\mathrm{E}_{x_{0}}\left[\hat{r}\left(\delta_{x_{0}}\right)\right]<\infty$, then $\mathrm{E}_{\pi}\left[r\left(\delta_{x_{0}}\right)\right]<\infty$.

Proof. By virtue of Theorem 2.1, if $\mathrm{E}_{x_{0}}\left[\hat{r}\left(\delta_{x_{0}}\right)\right]<\infty$ we know that there exists a skeleton chain $\Phi_{n h_{0}}$ such that $\mathrm{E}_{x_{0}}\left[r^{0}\left(\delta_{x_{0}}\left(h_{0}\right)\right)\right]<\infty$. It follows, from Theorem 2.6 of [15], that

$$
\mathrm{E}_{\pi}\left[r\left(\delta_{x_{0}}\left(h_{0}\right)\right)\right]<\infty .
$$

If $y$, the initial state of $\Phi_{t}$, does not equal $x_{0}$, then $\delta_{x_{0}} \leq \delta_{x_{0}}(h)$, meaning that we have

$$
\mathrm{E}_{y}\left[r\left(\delta_{x_{0}}\right)\right] \leq \mathrm{E}_{y}\left[r\left(\delta_{x_{0}}\left(h_{0}\right)\right)\right]
$$

for any $y \neq x_{0}$. Since $\mathrm{E}_{x_{0}}\left[\hat{r}\left(\delta_{x_{0}}\right)\right]<\infty$, it follows from (2.7) that $\mathrm{E}_{x_{0}}\left[r\left(\delta_{x_{0}}\right)\right]<\infty$, whence

$$
\begin{aligned}
\mathrm{E}_{\pi}\left[r\left(\delta_{x_{0}}\right)\right] & =\pi\left(x_{0}\right) \mathrm{E}_{x_{0}}\left[r\left(\delta_{x_{0}}\right)\right]+\int_{X \backslash\left\{x_{0}\right\}} \pi(\mathrm{d} y) \mathrm{E}_{y}\left[r\left(\delta_{x_{0}}\right)\right] \\
& \leq \pi\left(x_{0}\right) \mathrm{E}_{x_{0}}\left[r\left(\delta_{x_{0}}\right)\right]+\mathrm{E}_{\pi}\left[r\left(\delta_{x_{0}}\left(h_{0}\right)\right)\right]<\infty .
\end{aligned}
$$

Remark 2.4. Suppose that $r, \hat{r} \in \Lambda$. It follows from Theorem 2.3 that if $\mathrm{E}_{x_{0}}\left[\int_{0}^{\delta_{x_{0}}} \hat{r}(t) \mathrm{d} t\right]<\infty$, then $\mathrm{E}_{\pi}\left[\hat{r}\left(\delta_{x_{0}}\right)\right]<\infty$. Many subgeometric functions satisfy the required assumption. For example, let $r(t)=(l+1) t^{l}, l \in \mathbb{R}_{+}$. Then $\hat{r}(t)=t^{l+1}$, and it is obvious that $r, \hat{r} \in \Lambda$. 


\section{Length of the $M / G / 1$ queue with multiple vacations}

There is much literature (e.g. [2] and [9]) on M/G/1 queues with vacations. These queues include, for example, the $\mathrm{M} / \mathrm{G} / 1$ queue with step-up time, with $N$-policy, with single vacation, or with multiple vacations. In this section, we consider the most complicated case: the M/G/1 queue with multiple vacations, which is denoted simply by M/G/1(E, MV). The other queues can be studied more easily via the same method. For more details about M/G/1(E, MV), see [5], [7], and [10]. This queue is obtained by introducing the strategy of exhaustive service and multiple vacation to the classical M/G/1 queue: once the system has no customers, the server begins a vacation of random length $V$ immediately. If, when the vacation ends, the system still has no customers, the server continues with further independent, identically distributed vacations that do not end until the system has customers queueing when a vacation ends. Here $V$ is always assumed to be a nonnegative random variable, with distribution function $V(x)$, that has finite first and second moments, i.e. $\mathrm{E}[V]<\infty$ and $\mathrm{E}\left[V^{2}\right]<\infty$. For M/G/1(E, MV), the customers arrive according to a Poisson process with parameter $\lambda, 0<\lambda<\infty$, and the service time $B$ has a general distribution $B(x)$.

Let $L_{t}$ be the queue length process of M/G/1(E, MV). It is known that $L_{t}$ is not a Markov process unless $B(x)$ is exponentially distributed. We introduce a supplementary variable as follows:

$$
\begin{aligned}
\theta_{t} & =\text { the elapsed service time of the customer being served at time } t \\
& =0 \quad \text { if the server is idle at time } t
\end{aligned}
$$

Then $\left(L_{t}, \theta_{t}\right)$ becomes a continuous-time Markov process on the two-dimensional state space $X=\mathbb{Z}_{+} \times \mathbb{R}_{+}$. Several types of ergodicity for the discrete-time embedded chain of the queue length process $L(t)$ were studied in [8]. Here, we study the subgeometric convergence of the continuous-time queue length process $\left(L_{t}, \theta_{t}\right)$.

Let $Q_{\mathrm{b}}$ be the number of customers in the system when one busy period begins. Then

$$
\mathrm{P}\left[Q_{\mathrm{b}}=j\right]=\frac{v_{j}}{1-v_{0}}, \quad j \in \mathbb{N}_{+},
$$

where

$$
v_{j}=\int_{0}^{\infty} \frac{(\lambda t)^{j}}{j !} \mathrm{e}^{-\lambda t} \mathrm{~d} V(t), \quad j \in \mathbb{Z}_{+},
$$

is the probability that $j$ customers join the queue during a vacation.

Denote by $D_{v}$ the busy period of M/G/1(E, MV) and by $D$ the busy period of the classical $\mathrm{M} / \mathrm{G} / 1$ queue. It is easy to see that

$$
\left\{D_{v} \mid Q_{\mathrm{b}}=k\right\}=\left\{D_{1}+D_{2}+\cdots+D_{k}\right\}
$$

where $D_{k}$ is the busy period of the classical M/G/1 queue caused by the $k$ th customer, and the $D_{i}$ are independent and identically distributed. Let $J$ be the number of vacations during a series of consecutive vacations. Then

$$
\mathrm{P}[J=j]=v_{0}^{j-1}\left(1-v_{0}\right), \quad j \in \mathbb{N}_{+} .
$$

Let $V_{v}$ be the vacation period of M/G/1(E, MV). Then

$$
\left\{V_{v} \mid J=j\right\}=\left\{V_{1}+V_{2}+\cdots+V_{j}\right\}
$$


where $V_{i}$ denotes the $i$ th vacation and the $V_{i}$ are independent and identically distributed. Define $1 / \mu=\int_{0}^{\infty} x \mathrm{~d} B(x)$ and $\rho=\lambda / \mu$. From the known result $\mathrm{E}[D]=1 / \mu(1-\rho)$, we have

$$
\mathrm{E}\left[D_{v}\right]=\frac{\rho \mathrm{E}[V]}{(1-\rho)\left(1-v_{0}\right)}, \quad \mathrm{E}\left[V_{v}\right]=\frac{\mathrm{E}[V]}{1-v_{0}} .
$$

The following proposition is a known result [17], which is adopted to study the Harris ergodicity of the queue length process.

Proposition 3.1. Let $\Phi_{n}$ be a discrete-time Markov chain on a general state space X. Suppose that there exists a state $x_{0} \in X$ such that

(i) $\operatorname{gcd}\left\{n \geq 1: P^{n}\left(x_{0},\left\{x_{0}\right\}\right)>0\right\}=1$ (where gcd stands for 'greatest common divisor');

(ii) $\inf _{x \in X} \mathrm{P}_{x}\left[S_{x_{0}}<\infty\right]>0$, where $S_{x_{0}}=\inf \left\{n \geq 1: \Phi_{n}=x_{0}\right\}$; and

(iii) $\mathrm{E}_{x_{0}}\left[S_{x_{0}}\right]<\infty$.

Then $\Phi_{n}$ is Harris ergodic.

It is easy to see that when $\left(L_{t}, \theta_{t}\right)$ hits the state $(0,0)$, it will stay there for a random length of time that is exponentially distributed with parameter $\lambda$, meaning that $\left(L_{t}, \theta_{t}\right)$ satisfies Assumption 1.1. We first study the Harris ergodicity of $\left(L_{t}, \theta_{t}\right)$ and then investigate the subgeometric rates of convergence based on Harris ergodicity.

Theorem 3.1. For $M / G / 1(E, M V),\left(L_{t}, \theta_{t}\right)$ is Harris ergodic if and only if $\rho<1$.

Proof. Suppose that $\rho<1$. From Lemma 2.1, we only need to prove that some skeleton chain $\left(L_{n h_{0}}, \theta_{n h_{0}}\right)$ is Harris ergodic.

It follows from

$$
P^{n h}((0,0),\{(0,0)\}) \geq \mathrm{e}^{-\lambda n h}, \quad n \in \mathbb{N}_{+},
$$

that part (i) of Proposition 3.1 holds. Since $\rho<1$ the system is stable, meaning that

$$
\mathrm{P}_{(i, x)}\left\{\left(L_{n h}, \theta_{n h}\right)=(0,0), \text { i.o. }\right\}=1
$$

for any $(i, x) \in \mathbb{Z}_{+} \times \mathbb{R}_{+}$, where 'i.o.' means 'infinitely often'. Hence, part (ii) of Proposition 3.1 holds.

From (3.1), we know that if $\rho<1$, then $\mathrm{E}_{(0,0)}\left[\delta_{(0,0)}\right]=\mathrm{E}\left[D_{v}\right]+\mathrm{E}\left[V_{v}\right]<\infty$. It follows from Remark 2.1 that $\mathrm{E}_{(0,0)}\left[\delta_{(0,0)}\left(h_{0}\right)\right]<\infty$ for some $h_{0}>0$. Thus, part (iii) of Proposition 3.1 holds, $\left(L_{n h_{0}}, \theta_{n h_{0}}\right)$ is Harris ergodic, and, by Lemma 2.1 , if follows immediately that $\left(L_{t}, \theta_{t}\right)$ is Harris ergodic.

On the other hand, suppose that $\left(L_{t}, \theta_{t}\right)$ is Harris ergodic. Then, for any $h>0,\left(L_{n h}, \theta_{n h}\right)$ is also Harris ergodic. Since $\{(0,0)\}$ is a small set for $\left(L_{n h}, \theta_{n h}\right)$, we have $\mathrm{E}_{(0,0)}\left[\delta_{(0,0)}(h)\right]<\infty$ and it follows, from Remark 2.1, that $\mathrm{E}_{(0,0)}\left[\delta_{(0,0)}\right]<\infty$. From (3.1), we find that $\rho<1$.

Theorem 3.2. Let $l \in \mathbb{R}_{+}$such that $l \geq 1$. For the process $\left(L_{t}, \theta_{t}\right)$ of $M / G / 1(E, M V)$, $\mathrm{E}_{(0,0)}\left[\delta_{(0,0)}^{l}\right]<\infty$ if and only if $\int_{0}^{\infty} t^{l} \mathrm{~d} V \overline{(t)}<\infty$ and $\int_{0}^{\infty} t^{l} \mathrm{~d} B(t)<\infty$.

Proof. For a $k \in \mathbb{Z}_{+}$such that $k \geq 2$, we have

$$
k^{l}=[(k-1)+1]^{l} \leq \max \left\{1,2^{l-1}\right\}\left[(k-1)^{l}+1\right]=2^{l-1}\left[(k-1)^{l}+1\right] \leq 2^{l}(k-1)^{l} .
$$


For such a $k$, using the above inequality we have

$$
\begin{aligned}
\sum_{k=l+1}^{\infty} k^{l} \frac{(\lambda t)^{k}}{k !} & =\sum_{k=l+1}^{\infty} k^{l-1} \frac{(\lambda t)^{k-1}}{(k-1) !} \lambda t \\
& \leq \sum_{k=l+1}^{\infty} 2^{l-1}(k-1)^{l-1} \frac{(\lambda t)^{k-1}}{(k-1) !} \lambda t \\
& \leq \sum_{k=l-1}^{\infty} 2^{l-1} 2^{l-2} k^{l-2} \frac{(\lambda t)^{k}}{k !}(\lambda t)^{2} \\
& \leq \sum_{k=1}^{\infty} 2^{l-1} 2^{l-2} \cdots 2^{l-l} k^{l-l} \frac{(\lambda t)^{k}}{k !}(\lambda t)^{l} \\
& =\sum_{k=1}^{\infty} 2^{l(l-1) / 2} \frac{(\lambda t)^{k}}{k !}(\lambda t)^{l} .
\end{aligned}
$$

Let $\left\{u_{i}, i \in \mathbb{N}_{+}\right\}$be a sequence of nonnegative real numbers. For a $p \in \mathbb{R}_{+}$such that $p \geq 1$, by using Jensen's inequality on the convex function $x \rightarrow x^{p}, x \geq 0$ we see that

$$
\left(\frac{1}{n} \sum_{i=1}^{n} u_{i}\right)^{p} \leq \frac{1}{n} \sum_{i=1}^{n} u_{i}^{p}
$$

and, thus,

$$
\left(\sum_{i=1}^{n} u_{i}\right)^{p} \leq n^{p-1} \sum_{i=1}^{n} u_{i}^{p} .
$$

Many research works study the moments of busy period $D$ in the classical M/G/1 queue. From Theorem 2.1 of [6] or Example 1 of [4], we know that $\mathrm{E}\left[D^{l}\right]<\infty$ if and only if $\int_{0}^{\infty} t^{l} \mathrm{~d} B(t)<\infty$.

We first prove the sufficiency hypothesis. If $\int_{0}^{\infty} t^{l} \mathrm{~d} V(t)<\infty$ and $\int_{0}^{\infty} t^{l} \mathrm{~d} B(t)<\infty$ then, from (3.2) and (3.3), we obtain

$$
\begin{aligned}
\mathrm{E}\left[D_{v}^{l}\right] & =\sum_{k=1}^{\infty} \mathrm{P}\left[Q_{\mathrm{b}}=k\right] \mathrm{E}\left[\left(D_{1}+D_{2}+\cdots+D_{Q_{\mathrm{b}}}\right)^{l} \mid Q_{\mathrm{b}}=k\right] \\
& \leq \sum_{k=1}^{\infty} \frac{v_{k}}{1-v_{0}} k^{l} \mathrm{E}\left[D^{l}\right] \\
& =\frac{\mathrm{E}\left[D^{l}\right]}{1-v_{0}} \sum_{k=1}^{\infty} \int_{0}^{\infty} k^{l} \frac{(\lambda t)^{k}}{k !} \mathrm{e}^{-\lambda t} \mathrm{~d} V(t) \\
& =\frac{\mathrm{E}\left[D^{l}\right]}{1-v_{0}}\left[\sum_{k=1}^{l} \int_{0}^{\infty} k^{l} \frac{(\lambda t)^{k}}{k !} \mathrm{e}^{-\lambda t} \mathrm{~d} V(t)+\sum_{k=l+1}^{\infty} \int_{0}^{\infty} k^{l} \frac{(\lambda t)^{k}}{k !} \mathrm{e}^{-\lambda t} \mathrm{~d} V(t)\right] \\
& \leq \frac{\mathrm{E}\left[D^{l}\right]}{1-v_{0}}\left(l^{l}+2^{l(l-1) / 2} \lambda^{l} \mathrm{E}\left[V^{l}\right]\right) \\
& <\infty
\end{aligned}
$$


Similarly, we have

$$
\begin{aligned}
\mathrm{E}\left[V_{v}^{l}\right] & =\sum_{j=1}^{\infty} \mathrm{P}[J=j] \mathrm{E}\left[\left(V_{1}+V_{2}+\cdots+V_{J}\right)^{l} \mid J=j\right] \\
& \leq \sum_{j=1}^{\infty}\left(1-v_{0}\right) v_{0}^{j-1} j^{l} \mathrm{E}\left[V^{l}\right] \\
& <\infty
\end{aligned}
$$

Hence,

$$
\mathrm{E}_{(0,0)}\left[\delta_{(0,0)}^{l}\right]=\mathrm{E}\left[\left(D_{v}+V_{v}\right)^{l}\right] \leq \max \left\{1,2^{l-1}\right\}\left(\mathrm{E}\left[D_{v}^{l}\right]+\mathrm{E}\left[V_{v}^{l}\right]\right)<\infty .
$$

Now we prove the necessity hypothesis. If $\mathrm{E}_{(0,0)}\left[\delta_{(0,0)}^{l}\right]<\infty$ then, owing to the fact that

$$
\mathrm{E}_{(0,0)}\left[\delta_{(0,0)}^{l}\right]=\mathrm{E}\left[\left(D_{v}+V_{v}\right)^{l}\right] \geq \mathrm{E}\left[D_{v}^{l}\right]+\mathrm{E}\left[V_{v}^{l}\right],
$$

we find that $\int_{0}^{\infty} t^{l} \mathrm{~d} V(t)<\infty$ and $\int_{0}^{\infty} t^{l} \mathrm{~d} B(t)<\infty$, as required.

Let $r(t)=t^{l}$ for $l \in \mathbb{R}_{+}$such that $l \geq 1$. From Theorem 2.2 and Theorem 3.2, we have the following result.

Theorem 3.3. Suppose that the process $\left(L_{t}, \theta_{t}\right)$ of $M / G / 1(E, M V)$ is Harris ergodic. If

$$
\int_{0}^{\infty} t^{l} \mathrm{~d} V(t)<\infty \text { and } \int_{0}^{\infty} t^{l} \mathrm{~d} B(t)<\infty,
$$

then

$$
t^{l-1}\left\|P^{t}((i, x), \cdot)-\pi(\cdot)\right\| \rightarrow 0, \quad t \rightarrow \infty
$$

for $\pi$-a.e. $(i, x) \in X$.

\section{Explicit rates of convergence for stochastically ordered Markov processes}

In this section, we suppose that $\Phi_{t}$ is a pathwise-ordered Markov process on the state space $X=[0, \infty)$; i.e. a sample path of the process with a high initial state is never below a sample path of the process with a lower initial state. The 'taboo' probability of being in the set $A$ at time $t$ without first passing through $\{0\}$ is

$$
{ }_{0} P^{t}(x, A)=\mathrm{P}_{x}\left[\left\{\Phi_{t} \in A\right\} \cap\left\{\delta_{0} \geq t\right\}\right]
$$

for $x>0$. We will make the irreducibility assumption that, for some $\eta>0$, the process can travel from 0 to $[\eta, \infty)$ with positive probability before first returning to 0 , and, for each $y$, $y>x>0$, can travel from $x$ to $[y, \infty)$ without passing through $\{0\}$; i.e. there exist $t_{1}, t_{2}>0$ such that

$$
{ }_{0} P^{t_{1}}(0,[\eta, \infty))>0, \quad{ }_{0} P^{t_{2}}(x,[y, \infty))>0 .
$$

For a continuous-time Markov process as described above, the exponential convergence rates were found explicitly in [11] using the coupling method. We aim to apply the coupling method here to give explicit bounds on the subgeometric rates of convergence. The following lemma establishes a property of stochastically ordered Markov processes. 
Lemma 4.1. Let $\Phi_{t}$ be a stochastically ordered Markov process satisfying (4.1). If $r \in \Lambda$ then $\mathrm{E}_{0}\left[\hat{r}\left(\delta_{0}\right)\right]<\infty$ if and only if $\mathrm{E}_{x}\left[\hat{r}\left(\delta_{0}\right)\right]<\infty$ for every $x>0$.

Proof. Obviously $\hat{r}(t)$ is nondecreasing in $t$, so the pathwise ordering of $\Phi_{t}$ shows that $\mathrm{E}_{x}\left[\hat{r}\left(\delta_{0}\right)\right]$ is nondecreasing in $x$. Hence, if $\mathrm{E}_{x}\left[\hat{r}\left(\delta_{0}\right)\right]<\infty$ for some $x>0$ then $\mathrm{E}_{y}\left[\hat{r}\left(\delta_{0}\right)\right] \leq$ $\mathrm{E}_{x}\left[\hat{r}\left(\delta_{0}\right)\right]<\infty$ for each $y<x$. Let $y>x$ and choose a $t_{2}>0$ such that ${ }_{0} P^{t_{2}}(x,[y, \infty))>0$. By the monotonicity of $\mathrm{E}_{x}\left[\hat{r}\left(\delta_{0}\right)\right]$ in $x$, we have

$$
\mathrm{E}_{x}\left[\hat{r}\left(\delta_{0}\right)\right] \geq \int_{y}^{\infty}{ }_{0} P^{t_{2}}(x, \mathrm{~d} z) \mathrm{E}_{z}\left[\hat{r}\left(\delta_{0}\right)\right] \geq \mathrm{E}_{y}\left[\hat{r}\left(\delta_{0}\right)\right]_{0} P^{t_{2}}(0,[y, \infty)) .
$$

Thus, we have proved that $\mathrm{E}_{x}\left[\hat{r}\left(\delta_{0}\right)\right]<\infty$ for some $x>0$ if and only if $\mathrm{E}_{x}\left[\hat{r}\left(\delta_{0}\right)\right]<\infty$ for every $x>0$.

Now suppose that $\mathrm{E}_{0}\left[\hat{r}\left(\delta_{0}\right)\right]<\infty$ and use the irreducibility assumption to choose a $t_{1}>0$ such that ${ }_{0} P^{t_{1}}(x,[y, \infty))>0$. By the monotonicity of $\mathrm{E}_{x}\left[\hat{r}\left(\delta_{0}\right)\right]$ in $x$, we now have

$$
\mathrm{E}_{0}\left[\hat{r}\left(\delta_{0}\right)\right] \geq \int_{\eta}^{\infty}{ }_{0} P^{t_{1}}(0, \mathrm{~d} z) \mathrm{E}_{z}\left[\hat{r}\left(\delta_{0}\right)\right] \geq \mathrm{E}_{\eta}\left[\hat{r}\left(\delta_{0}\right)\right]_{0} P^{t_{1}}(0,[\eta, \infty)) .
$$

Thus, if $\mathrm{E}_{0}\left[\hat{r}\left(\delta_{0}\right)\right]<\infty$ then $\mathrm{E}_{\eta}\left[\hat{r}\left(\delta_{0}\right)\right]<\infty$ for some $\eta>0$, meaning that $\mathrm{E}_{x}\left[\hat{r}\left(\delta_{0}\right)\right]<\infty$ for every $x>0$.

We introduce further notation for the first hitting time as follows: $\tau_{0}=\inf \left\{t \geq 0: \Phi_{t}=0\right\}$. Note the relationships between $\delta_{0}$ and $\tau_{0}$ : when $\Phi_{0}=0$ we have $\tau_{0}=0 \neq \delta_{0}$, and when $\Phi_{0} \neq 0$ we have $\tau_{0}=\delta_{0}$.

Theorem 4.1. Let $\Phi_{t}$ be a stochastically ordered Markov process satisfying Assumption 1.1 and (4.1). If $r, \hat{r} \in \Lambda$ and $\mathrm{E}_{0}\left[\int_{0}^{\delta_{0}} \hat{r}(t) \mathrm{d} t\right]<\infty$, then

$$
\int_{0}^{\infty} r(t)\left\|P^{t}(x, \cdot)-\pi(\cdot)\right\| \mathrm{d} t \leq M(x)
$$

for every $x \geq 0$, where

$$
M(x)=2 \mathrm{E}_{x}\left[\hat{r}\left(\tau_{0}\right)\right]+2 \mathrm{E}_{\pi}\left[\hat{r}\left(\tau_{0}\right)\right]<\infty .
$$

Proof. Let $\Phi_{t}^{1}$ and $\Phi_{t}^{2}$ be two copies of the process, with the initial conditions $\Phi_{0}^{1} \equiv x$ and $\Phi_{0}^{2} \equiv Y$, where $Y$ is a random variable on $(\Omega, \mathcal{F}, \mathrm{P})$ with invariant probability measure $\pi$. We define $T=\inf \left\{t \geq 0: \Phi_{t}^{1}=\Phi_{t}^{2}\right\}$ to be the coupling time and use the coupling inequality to find that

$$
\left\|P^{t}(x, \cdot)-\pi(\cdot)\right\| \leq 2 \mathrm{P}_{x, \pi}[T>t] .
$$

Owing to the path-wise ordering of $\Phi_{t}$, we have $\mathrm{P}_{x, \pi}[T>t] \leq \mathrm{P}_{v}\left[\tau_{0}>t\right]$, where $v(A)=$ $\mathrm{P}[\max \{Y, x\} \in A]$ for $A \in \mathscr{B}(X)$. Hence, by virtue of the Markov inequality and (4.2) we obtain

$$
\begin{aligned}
\left\|P^{t}(x, \cdot)-\pi(\cdot)\right\| & \leq 2 \mathrm{P}_{v}\left[\tau_{0}>t\right] \\
& =2 \mathrm{P}[Y \leq x] \mathrm{P}_{x}\left[\tau_{0}>t\right]+2 \int_{x}^{\infty} \mathrm{P}_{u}\left[\tau_{0}>t\right] \pi(\mathrm{d} u) \\
& \leq 2 \mathrm{P}_{x}\left[\tau_{0}>t\right]+2 \mathrm{P}_{\pi}\left[\tau_{0}>t\right] .
\end{aligned}
$$


Since $\mathrm{E}_{0}\left[\int_{0}^{\delta_{0}} \hat{r}(t) \mathrm{d} t\right]<\infty$, we have $\mathrm{E}_{0}\left[\hat{r}\left(\tau_{0}\right)\right] \leq \mathrm{E}_{0}\left[\hat{r}\left(\delta_{0}\right)\right]<\infty$. By applying Lemma 4.1, we find that $\mathrm{E}_{x}\left[\hat{r}\left(\tau_{0}\right)\right] \leq \mathrm{E}_{x}\left[\hat{r}\left(\delta_{0}\right)\right]<\infty$ for every $x \in X$. From Remark 2.4, we infer that $\mathrm{E}_{\pi}\left[\hat{r}\left(\tau_{0}\right)\right] \leq \mathrm{E}_{\pi}\left[\hat{r}\left(\delta_{0}\right)\right]<\infty$. Hence,

$$
\begin{aligned}
\int_{0}^{\infty} r(t)\left\|P^{t}(x, \cdot)-\pi(\cdot)\right\| \mathrm{d} t & \leq 2 \int_{0}^{\infty} r(t) \mathrm{P}_{x}\left[\tau_{0}>t\right] \mathrm{d} t+2 \int_{0}^{\infty} r(t) \mathrm{P}_{\pi}\left[\tau_{0}>t\right] \mathrm{d} t \\
& =2 \mathrm{E}_{x}\left[\hat{r}\left(\tau_{0}\right)\right]+2 \mathrm{E}_{\pi}\left[\hat{r}\left(\tau_{0}\right)\right] \\
& =M(x) \\
& <\infty
\end{aligned}
$$

for every $x \in X$. Thus, our assertion holds.

\section{Waiting time of the $M / G / 1$ queue}

Let $W_{t}$ be the virtual waiting time of a customer who joins an M/G/1 queue at time $t$ [17], [11]. The sufficient and necessary conditions for Harris ergodicity and exponential ergodicity were found in [17], and the best exponential convergence rates were investigated in [11]. It is known that $W_{t}$ is stochastically ordered and that (4.1) holds. For an M/G/1 queue, the customers arrive according to a Poisson process with some parameter $\lambda$. Once $W_{t}$ hits the state 0 , it will stay there for a random length of time that is exponentially distributed. Thus, $W_{t}$ satisfies Assumption 1.1.

Theorem 5.1. If the virtual waiting time $W_{t}$ of an $M / G / 1$ queue is Harris ergodic then, for $l \in \mathbb{R}_{+}, \mathrm{E}_{0}\left[\tau_{0}^{l}\right]<\infty$ if and only if $\int_{0}^{\infty} t^{l} \mathrm{~d} B(t)<\infty$. Moreover, if $\int_{0}^{\infty} t^{l} \mathrm{~d} B(t)<\infty$ for some $l \geq 2$ then, for every $x \in X$,

$$
\int_{0}^{\infty} t^{l-2}\left\|P^{t}(x, \cdot)-\pi(\cdot)\right\| \mathrm{d} t \leq M(x),
$$

where

$$
M(x)=\mathrm{E}_{x}\left[\tau_{0}^{l-1}\right]+\mathrm{E}_{\pi}\left[\tau_{0}^{l-1}\right]<\infty .
$$

Proof. We know the result that $\mathrm{E}\left[D^{l}\right]<\infty$ if and only if $\int_{0}^{\infty} t^{l} \mathrm{~d} B(t)<\infty$. Since $\mathrm{E}_{0}\left[\tau_{0}^{l}\right]=$ $\mathrm{E}\left[D^{l}\right]$, it follows that $\mathrm{E}_{0}\left[\tau_{0}^{l}\right]<\infty$ if and only if $\int_{0}^{\infty} t^{l} \mathrm{~d} B(t)<\infty$. Let $r(t)=t^{l}$ for $l \in \mathbb{R}_{+}$ such that $l \geq 2$. It follows from Theorem 4.1 that our assertion holds.

\section{Continuous-time birth-death chains}

Let $\Phi_{t}$ be a continuous-time irreducible birth-death chain on a countable space $X=\mathbb{Z}_{+}$ with $Q$-matrix as follows:

$$
q_{i, i+1}=b_{i}, \quad i \in \mathbb{Z}_{+} ; \quad q_{i, i-1}=a_{i}, \quad i \in \mathbb{N}_{+} ; \quad q_{i j}=0, \quad|i-j| \geq 2 .
$$

We suppose that the $Q$-matrix is conservative and totally stable. From [19], we see that $\Phi_{t}$ is stochastically ordered. It is obvious that (4.1) and Assumption 1.1 hold. Suppose that $\Phi_{t}$ is ergodic. Then the invariant probability measure $\pi$ exists and can be computed as follows:

$$
\pi=\frac{\mu_{i}}{\mu}, \quad \mu=\sum_{i=0}^{\infty} \mu_{i}, \quad \mu_{0}=1, \quad \mu_{i}=\frac{b_{0} b_{1} \cdots b_{i-1}}{a_{1} a_{2} \cdots a_{i}}, \quad i \in \mathbb{N}_{+} .
$$


Let $r(t)=t^{l}$ for $l \in \mathbb{N}_{+}$. According to Theorem 1.4 of [12], we can compute $\mathrm{E}_{i}\left[\tau_{0}^{l}\right]$ as follows:

$$
\begin{aligned}
\mathrm{E}_{i}\left[\tau_{0}^{1}\right] & =\sum_{j=0}^{i-1} \frac{1}{\mu_{j} b_{j}} \sum_{k=j+1}^{\infty} \mu_{k} \quad \text { for every } i \in \mathbb{N}_{+}, \\
\mathrm{E}_{i}\left[\tau_{0}^{n+1}\right] & =n \sum_{j=0}^{i-1} \frac{1}{\mu_{j} b_{j}} \sum_{k=j+1}^{\infty} \mu_{k} \mathrm{E}_{k}\left[\left(\tau_{0}\right)^{n}\right] \quad \text { for } n \in \mathbb{N}_{+} .
\end{aligned}
$$

Since $\pi$ and $\mathrm{E}_{i}\left[\tau_{0}^{1}\right]$ can be computed completely, so too can the bound $M(i)$.

Theorem 6.1. Let $\Phi_{t}$ be an irreducible, regular birth-death chain. For $l \in \mathbb{Z}_{+}$such that $l \geq 2$, if $\mathrm{E}_{0}\left[\delta_{0}^{l}\right]<\infty$ then, for any $i \in X$,

$$
t^{l-1}\left|P^{t}(i, j)-\pi_{j}\right| \rightarrow 0, \quad t \rightarrow \infty
$$

Moreover,

$$
\int_{0}^{\infty} t^{l-2}\left|P^{t}(i, j)-\pi_{j}\right| \mathrm{d} t \leq M(i),
$$

where $M(i)=\mathrm{E}_{i}\left[\tau_{0}^{l-1}\right]+\sum_{i=0}^{\infty} \pi_{i} \mathrm{E}_{i}\left[\tau_{0}^{l-1}\right]<\infty$ and $\mathrm{E}_{0}\left[\tau_{0}^{l-1}\right]=0$.

The following example, in which we can compute the quantities $\mathrm{E}_{i}\left[\tau_{0}^{l-1}\right]$ and $\mathrm{E}_{\pi}\left[\tau_{0}^{l-1}\right]$, is taken from [12].

Example 6.1. Let $a_{i}=b_{i}=i^{\gamma}, \gamma \in(1,2]$. For any $l \geq 1$, if $\gamma>2-1 / l$ then from [12] we see that

$$
\mathrm{E}_{0}\left[\delta_{0}^{l}\right]<\infty \quad \text { and } \quad \mathrm{E}_{i}\left[\tau_{0}^{l-1}\right] \sim \sum_{j=0}^{i-1} j^{2(l-1)-1-l \gamma} .
$$

Hence, from Theorem 6.1, we have

$$
\int_{0}^{\infty} t^{l-2}\left|P^{t}(i, j)-\pi_{j}\right| \mathrm{d} t \leq \mathrm{E}_{i}\left[\tau_{0}^{l-1}\right]+\mathrm{E}_{\pi}\left[\tau_{0}^{l-1}\right]<\infty,
$$

where

$$
\mathrm{E}_{i}\left[\tau_{0}^{l-1}\right] \sim \sum_{j=0}^{i-1} j^{2(l-1)-1-l \gamma}, \quad i \in \mathbb{N}_{+}, \quad \mathrm{E}_{0}\left[\tau_{0}^{l-1}\right]=0,
$$

and

$$
\mathrm{E}_{\pi}\left[\tau_{0}^{l-1}\right] \sim \sum_{j=0}^{\infty} j^{2(l-1)-1-l \gamma} \frac{\sum_{i=j+1}^{\infty} i^{-\gamma}}{\sum_{i=0}^{\infty} i^{-\gamma}}
$$

\section{Acknowledgements}

We would like to thank the referees for helpful suggestions. The work of Hou and Liu is supported by the National Natural Science Foundation of China (grant no. 10171009). The work of Zhang is supported by the Australian Research Council Centre of Excellence for Mathematics and Statistics of Complex Systems. 


\section{References}

[1] Chen, M. F. (1992). From Markov Chains to Nonequilibrium Particle Systems. World Scientific, River Edge, NJ.

[2] Doshi, B. (1985). An M/G/1 queue with variable vacations. In Proc. Internat. Conf. Model. Techniques Tools Performance Anal., North-Holland, Amsterdam, pp. 67-81.

[3] Down, D., Meyn, S. P. And Tweedie, R. L. (1995). Exponential and uniform ergodicity of Markov processes. Ann. Prob. 23, 1671-1691.

[4] Foss, S. And SApozhnikov, A. (2004). On the existence of moments for the busy period in a single-server queue. Math. Operat. Res. 29, 592-601.

[5] Fuhrman, S. (1984). A note on the M/G/1 queue with server vacations. Operat. Res. 32, 1368-1373.

[6] Gut, A. (1974). On the moments of some first passage times for sums of dependent random variables. Stoch. Process. Appl. 2, 115-126.

[7] Harris, C. and Marchal, W. (1988). State dependence in M/G/1 server vacation models. Operat. Res. 36, $560-565$.

[8] Hou, Z. And LiU, Y. (2004). Explicit criteria for several types of ergodicity of the embedded M/G/1 and GI/M/n queues. J. Appl. Prob. 41, 778-790.

[9] Levy, H. AND Kleinrock, L. (1986). A queue with starter and a queue with vacations: delay analysis by decompostition. Operat. Res. 34, 426-436.

[10] Levy, Y. and Yechiali, U. (1975). Utilization of idle time in an M/G/1 queueing system. Manag. Sci. 22, 202-211.

[11] Lund, R. B., Meyn, S. P. and Tweedie, R. L. (1996). Computable exponential convergence rates for stochastically ordered Markov processes. Ann. Appl. Prob. 6, 218-237.

[12] Mao, Y. H. (2004). Ergodic degrees for continuous-time Markov chains. Sci. China A 47, 161-174.

[13] Meyn, S. P. ANd Tweedie, R. L. (1993). Stability of Markovian processes. II. Continuous-time processes and sampled chains. Adv. Appl. Prob. 25, 487-517.

[14] Meyn, S. P. And Tweedie, R. L. (1993). Stability of Markovian processes. III. Foster-Lyapunov criteria for continuous-time processes. Adv. Appl. Prob. 25, 518-548.

[15] Nummelin, E. ANd Tuominen, P. (1983). The rate of convergence in Orey's theorem for Harris recurrent Markov chains with applications to renewal theory. Stoch. Process. Appl. 15, 295-311.

[16] Thorisson, H. (1985). The queue GI/G/1: finite moments of the cycle variables and uniform rates of convergence. Stoch. Process. Appl. 19, 85-99.

[17] Tuominen, P. And Tweedie, R. L. (1979). Exponential ergodicity in Markovian queueing and dam models. $J$. Appl. Prob. 16, 867-880.

[18] Tuominen, P. And Tweedie, R. L. (1994). Subgeometric rates of convergence of $f$-ergodic Markov chains. Adv. Appl. Prob. 26, 775-798.

[19] Van Doorn, E. A. (1981). Stochastic Monotonicity and Queueing Applications of Birth-Death Processes (Lecture Notes Statist. 4). Springer, New York. 